\title{
Next Generation Marine Data Networks in an IoT Environment
}

\author{
Rabab Al-Zaidi, Member, IEEE \\ School of Computer Science and Electronic Engineering, \\ University of Essex, Colchester, UK. \\ AL-Mustansiriya University, College of Engineering, \\ Computer and Software Engineering Dept., Iraq. \\ Email: rjmohs@essex.ac.uk.
}

\author{
John Woods, Mohammed Al-Khalidi, Member, IEEE \\ Khattab M. Ali Alheeti, Member, IEEE, \\ and Klaus McDonald-Maier, Senior Member, IEEE \\ School of Computer Science and Electronic \\ Engineering, University of Essex, UK. \\ $\ddagger$ University of Anbar, College of Computer Anbar, Iraq.
}

\begin{abstract}
Packet data networks at sea offer the potential for increased safety, connectivity and meteorological data acquisition. Existing solutions including satellite communication are expensive and prohibitive to most small vessels. In this paper, an Internet of Things (IoT) application is proposed as a marine data acquisition and cartography system over Ship Ad-hoc Networks (SANET). Ships are proposed to communicate over Very High Frequency (VHF) which is already available on the majority of ships and are equipped with several sensors such as sea depth, temperature, wind speed and direction, etc. On shore, 5G base station nodes represent sinks for the collected data and are equipped with Mobile Edge Computing (MEC) capabilities for data aggregation and processing. The sensory data is ultimately aggregated at a central cloud on the internet to produce public up to date cartography systems. We discuss the deployment limitations and benefits of the proposed system and investigate it's performance using four different MANET routing protocols which are Ad hoc On-Demand Distance Vector (AODV), Ad hoc On-Demand Multipath Distance Vector (AOMDV), Destination-Sequenced Distance Vector (DSDV) and Dynamic Source Routing (DSR) protocols. Simulation results illustrate the efficiency of the proposed system with packet delivery rates of up to 60 percent at shore base stations.
\end{abstract}

Keywords- Mobile Edge Computing, Mobile Cloud, Ship Adhoc Networks, Mobile Ad-hoc Networks, Very High Frequency.

\section{INTRODUCTION}

A mobile Ad Hoc Network (MANET) is a system of mobile nodes which are connected by wireless links. Each of these nodes acts as a router and is free to move in any direction independently. MANETs are a popular telecommunication technology that can easily be applied to almost any environment having fast configuration and no need for any underlying infrastructures support. The popularity of MANETs is due to the wide range of available wireless services and increasing spread of communicating devices like cell phones, laptops, PDAs, etc., providing ubiquitous computing at low cost. Networks in the marine environment are not as mature as land-based wireless systems. Marine communication systems available today only provide the bare minimum essential services such as ship identification, positioning, location, course, heading, destination, tonnage, speed, etc... in the form of AIS (Automatic Identification System) using VHF radio frequencies. Inter ship satellite communication is possible but is a costly option when compared to conventional wireless communications and not affordable by most small to medium seagoing vessels [1].

In efforts to standardise VHF data network communication at sea, the international telecommunication union (ITU) has defined Recommendation ITU-R M.1842-1 "The Characteristics of VHF Radio Systems and Equipment for the Exchange of Data and Electronic Mail in the Maritime Mobile Service Radio Regularization (RR) Appendix 18 Channels" [2]. They have defined marine band VHF radio to operate on internationally agreed frequencies in the band from $156 \mathrm{MHz}$ to $163 \mathrm{MHz}$. They also provide a guideline on the use of digital technologies by VHF systems of different bandwidths [3]. As expansion of the $5 \mathrm{G}$ radio spectrum, Ofcom has allocated VHF spectrum for the Internet of Things (IoT), aiming to encourage Machine to Machine (M2M) applications to use spectrum that will enable them to connect wirelessly over distances that are not possible with other frequencies.

In this paper, we propose an Internet of Things (IoT) application as a marine data acquisition and cartography system over Ship Ad-hoc Networks (SANET). We extend our evaluation of the Ship Ad-hoc Network proposed in [4] [5] to IoT networks and discuss the limitations and benefits of the proposed application and network architecture. We use a model of the VHF radio that complies with the ITU standards for data communication in the marine environment to setup a physical layer in the NS2 simulator. We use Time division multiple access (TDMA) as the channel access method as proposed by the ITU for ship data communication over VHF channels. TDMA allows a number of users to use the same frequency channel by dividing the signal into several different time slots. The users transmit in rapid sequence, one by one, each using its unique time slot as shown in fig. 1. We investigate the performance of four different MANET routing protocols which are Ad hoc On-Demand Distance Vector (AODV), Ad hoc On-Demand Multi Path Distance Vector (AOMDV), Destination-Sequenced Distance Vector (DSDV) and Dynamic Source Routing (DSR) protocols. Simulation results illustrate the efficiency of the proposed system with packet delivery rates of up to 60 percent at shore base stations. 


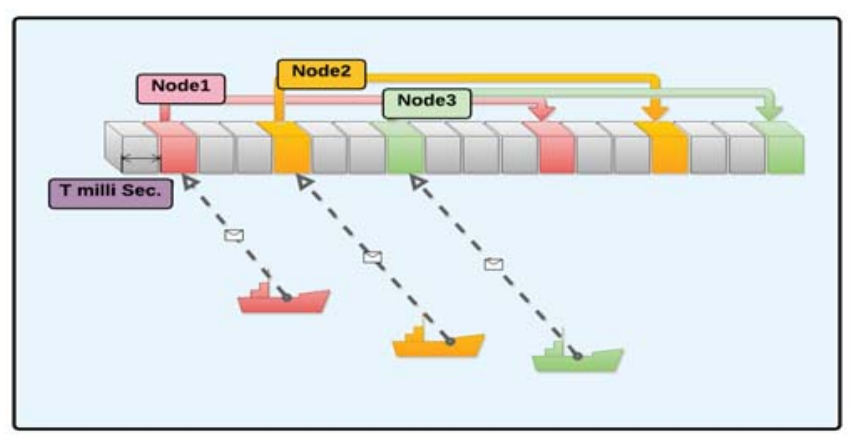

Fig. 1. TDMA Timeslot Allocation

The rest of the paper is structured as follows. Section 2 discusses the marine communication characteristics, while section 3 explains the proposed marine IoT system architecture. Section 4 provides an overview of the most popular routing protocols used in MANET's and thus in SANET's. Section 5 shows the simulation environment and Section 6 discusses the simulation results. The paper is finally concluded in section 7 .

\section{MARine Communication Characteristics}

When considering the establishment of packet networks in the marine environment a number of points come to mind. First of all, networks in the marine environment do share a number of characteristics and constraints with MANETs, VANETs(Vehicular ad hoc network) and other packet networks which effect the efficiency of network establishment and packet delivery ratio. And second marine networks also pose a series of unique characteristics of communication problems at sea that add a number of complexities and design constraints that are specific to ship ad hoc networking:

- First of all, marine networks are low bandwidth due to VHF channel limitations with packet data rates below 30 Kbps(Kilo bit per second).

- Power consumption is not an issue in marine communication as power is very low and available either from small batteries or using the ships power.

- Very large network areas covering vast oceans and seas, but with limited transmission ranges provided by VHF seldom reaching beyond $40 \mathrm{Km}$ for each ship.

- Marine networks are usually described as sparse networks comparing the number of ships at sea to the area the sea covers.

- Marine networks are weather governed networks as weather conditions will affect network density and mobility.

- Marine networks do not depend on any established infrastructure or centralized administration. Every node operates in a distributed peer-to-peer mode. Network management is distributed among different nodes, which brings added difficulty in fault detection and management.

- No central or default router available, each node acts as a router and forwards each others packets to enable information sharing between mobile hosts.

- In ship ad hoc networks, due to the nodes arbitrarily movement, the network topology, which is usually multihop, can change unpredictably, resulting in route changes, frequent network partitions, and possibly packet loss.

- Most network management algorithms were designed to work on fixed or relatively small wireless networks, while marine networks may involve tens of thousands of nodes. Scalability is essential for the successful deployment of these networks.

\section{Proposed Marine Iot System Architecture}

The emerging IoT is promising a fully connected world with an array of connected devices and services. VHF communication has been considered by Ofcom as one of the IoT enablers with the potential of providing a whole new set of services and applications that were not previously possible. And for that reason, Ofcom has added VHF to the $5 \mathrm{G}$ radio spectrum, setting the ground for $5 \mathrm{G}$ VHF connectivity. In this paper, we exploit this new $5 \mathrm{G}$ era to propose a cartography application where a SANET is used to collect different marine sensory data from ships and vessels and send this data back to onshore sinks collocated with 5G base station's that include dedicated storage as part of the mobile edge computing (MEG) services. We propose a new application of MEC where part of the edge computing resources is exploited as edge repositories (clouds) of collected sensory data that successfully arrives to shore. The edge clouds eventually connect to a central cloud in the internet where all the sensory data is aggregated, filtered and analysed to produce real-time maps of surface and under water environmental information that produces accumulative maps for beneficiary customers. The collected sensory data and map information can also be cached at network edges close to the users where high demand is observed by the network. The cartography system illustrated in fig. 2 can collect data including but not limited to: sea state, depth, temperature, wind speed/direction, humidity, salinity .. etc.

An identified obstacle is the bottleneck of traffic near the onshore sink. A quantization and compression method specific to marine sensory data has been proposed in our previous work [6] and partially alleviates this problem. For each of the sensors mentioned previously we have set the extreme lower and upper limits of the sensors readings likely to be found in the marine environment as well as the level of accuracy required to represent each reading. The predictability of gathered sensor data makes it beneficial to quantize the data to reduce the amount of bits needed to represent each reading in the binary representation. Applying this quantization in conjunction with the compression algorithm (AMDC) proposed in [6] has given effective data compression rates in comparison to the main compression methods. 


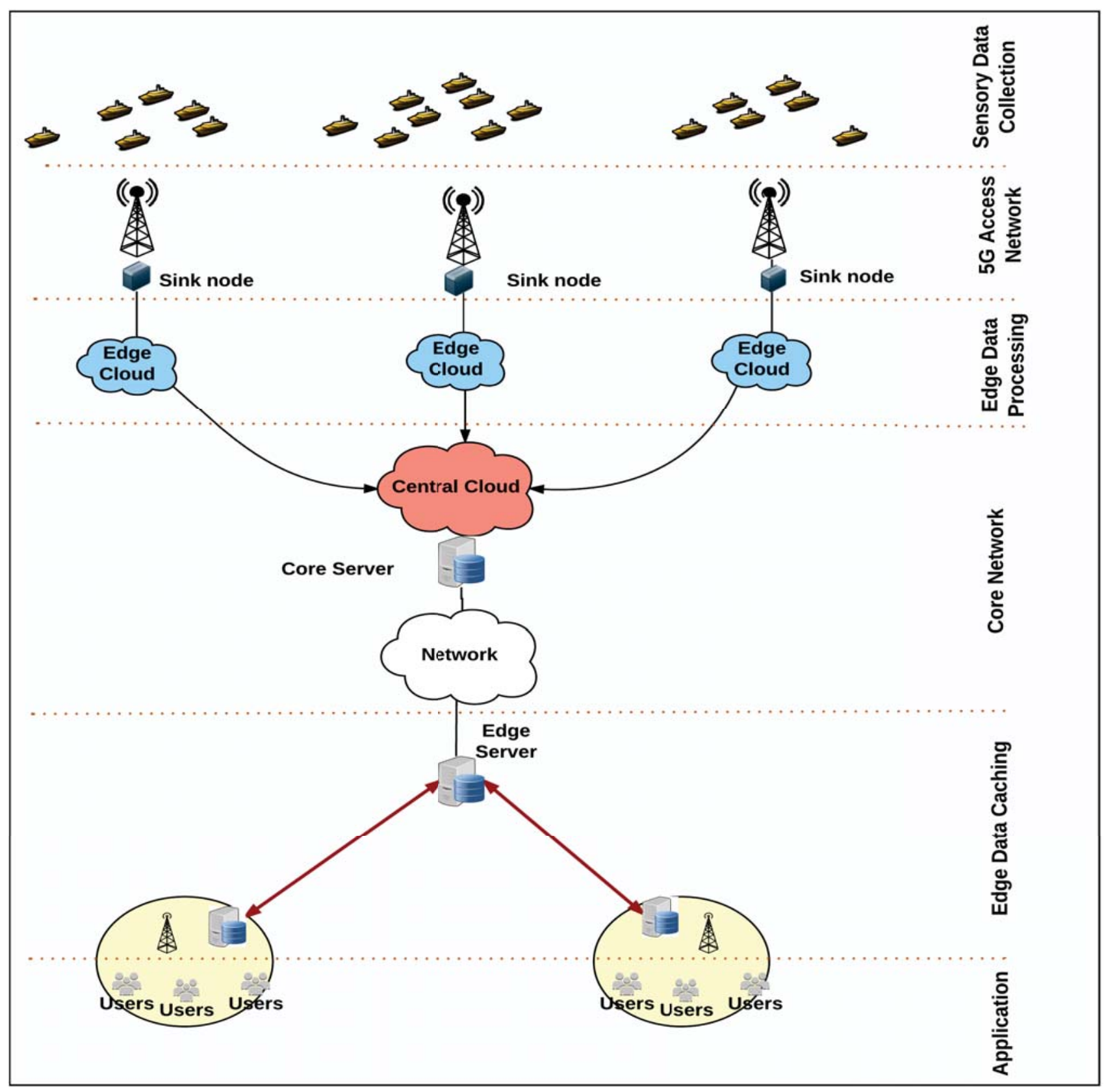

Fig. 2. Proposed Marine IoT System Architecture

\section{Routing Protocol Overview}

Routing protocols in MANET's and thus in SANET's can be classified into three main types according to the way routes are maintained in the network [7] as shown in fig. 3 .

\section{A. Proactive Routing Protocols}

Proactive or Table-Driven protocols maintain routing information even before this information is required. Each node maintains routing information to every other node in the network. Route information is generally stored in routing tables and is periodically updated with any change in the network topology. The protocols that fall under this category maintain different numbers of tables. Also, they are not suitable for large scale networks, because they need to maintain entries for each node in the routing table [8]. The DSDV proactive routing protocol will be used in our simulation.

\section{B. Reactive Routing Protocols (On-Demand)}

In reactive or on demand protocols, nodes initiate route discovery throughout the network, only when they want to 


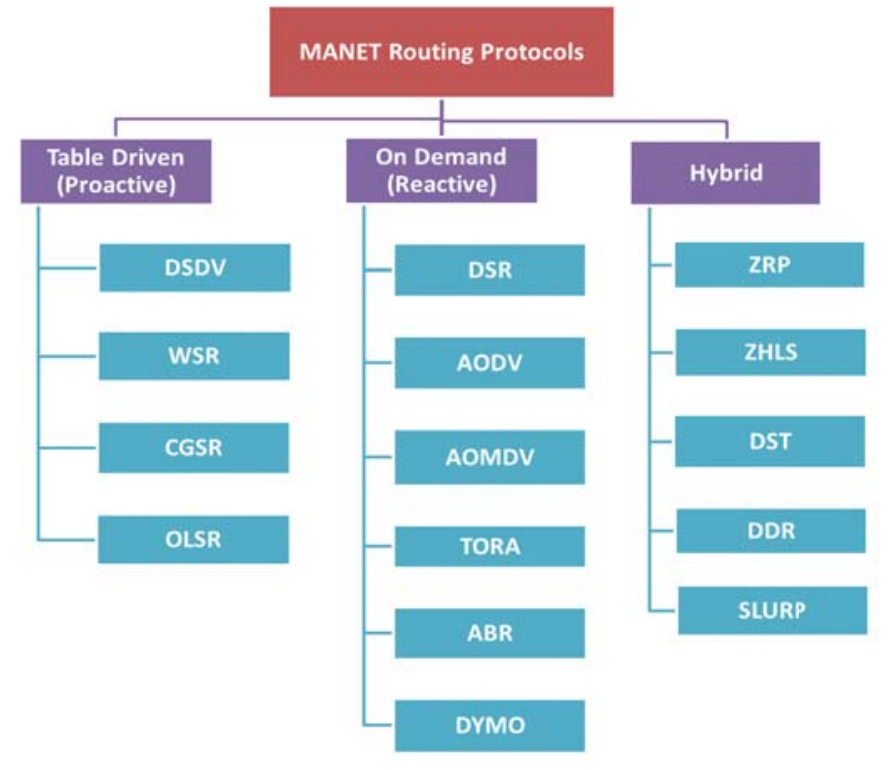

Fig. 3. MANET Routing Protocols

send packets to the destination. For this purpose, a node initiates a route discovery process through the network. The route discovery process is completed once a route is established or all possible variations have been examined. Once a route has been established, it is maintained by a route maintenance process until either the destination becomes inaccessible along every path from the source or until the route is no longer desired through the use of timers [7]. AODV, AOMDV and DSR reactive routing protocols will be used in our simulation.

\section{Hybrid Routing Protocols}

Hybrid routing protocols are a new protocol generation; they are together proactive and reactive in nature. These protocols are designed this way to increase their scalability by allowing the nodes that have close proximity to work together forming some sort of a backbone to reduce the route discovery overheads. This is accomplished by proactively maintaining routes to all nearby nodes and establishing routes to far away nodes using a route discovery strategy. The Majority of hybrid protocols proposed are zone-based, meaning that the network is partitioned or seen as a number of zones while others group nodes into trees or clusters [9]. Examples of these types of protocols are Zone routing protocol (ZRP) [10], Zone-based hierarchical link state (ZHLS) [11], Scalable location update routing protocol (SLURP), Distributed spanning trees based routing protocol (DST) [12], Distributed dynamic routing (DDR) [13] etc....

\section{Simulation EnVIRONMENT}

To evaluate the performance of the proposed marine network, we use a model of the VHF radio that complies with the ITU standards to setup a physical layer in the NS2 simulator (Network Simulator Version 2). We use Time division multiple access (TDMA) as the channel access method as proposed by the ITU for ship data communication over VHF channels. The
TABLE I

SIMULATION PARAMETERS.

\begin{tabular}{|c|c|}
\hline Indicator & Value \\
\hline Simulator & NS2.35 \\
\hline Routing Protocols & AODV, DSDV,AOMDV ,DSR \\
\hline Simulation Time & Fre00 sec \\
\hline Propagation model & Omni Antenna \\
\hline Antenna Model & Wireless channel \\
\hline Channel Type & CBR \\
\hline Traffic Type & $200 \mathrm{Km} \times 200 \mathrm{Km}$ \\
\hline No of Nodes & $30 \mathrm{Km}, 40 \mathrm{Km}$ \\
\hline Simulation Area & Random Way Point \\
\hline Transmission Range & $15 \mathrm{~m} / \mathrm{s}$ \\
\hline Mobility Model & $9.6 \mathrm{~Kb}$ \\
\hline Maximum speed of nodes &
\end{tabular}

TABLE II

HARDWARE AND OPERATING SYSTEM CONFIGURATION.

\begin{tabular}{|c|c|}
\hline Indicator & Description \\
\hline Processor & Core(TM)i73537U , CPU 2.50 GHz \\
\hline RAM & $8.00 \mathrm{~GB}$ \\
\hline OS & Ubuntu 12.04 \\
\hline Kernel & Linux \\
\hline System type & x64-based processor \\
\hline
\end{tabular}

traffic source type used in the simulation is CBR (Constant Bit Rate) traffic. The mobility model used to generate node movement is the Random Way Point model in a simulation area of $200 \mathrm{Km} \times 200 \mathrm{Km}$. The simulation was performed using four popular MANET routing protocols which are DSR, AODV, AOMDV and DSDV. For result resilience and accuracy we have run the simulation ten times and the average was extracted to analyse each performance factor for these four protocols. The number of CBR connections that were established in our simulation were 80 connections, the data packet size is 512 bits. Table I shows a summary of the simulation parameters used in our simulation. Also the hardware and operating system configuration used are listed in Table II

\section{RESUlts AND DisCUSSION}

A various number of metrics have been utilized to evaluate the performance of the routing protocols used in our network in terms of Normalized Routing Load, End-to-end Delay and Packet delivery ratio. Table III shown the comparison for those three metrics for each of the routing protocols used in the simulation.

\section{A. Normalized Routing Load}

Normalized Routing Load is the number of routing packets transmitted during the simulation. For packets sent over multiple hops, each transmission of the packet (each hop) counts as one transmission. In other words, it is a measure of the network load with control packets compared to the total number of sent data packets. It is desirable to have as few control packages as possible in order minimise energy use as in equation 1.

$$
R D=\frac{\sum \text { RoutingPackets }}{\sum \text { DataPacket }}
$$


TABLE III

COMPARISON OF AODV,AOMDV, DSDV AND DSR

\begin{tabular}{|c|c|c|c|c|}
\hline Algorithm Priority & AODV & AOMDV & DSDV & DSR \\
\hline Reactive & Yes & Yes & No & Yes \\
\hline Proactive & No & No & Yes & No \\
\hline Packet Delivery Ratio & Higer than DSDV & Highest & Lowest & Lower than AOMDV \\
\hline Normalized Routing Load & Best performance & Worst performance & Better than AOMDV & Better than AODV \\
\hline End To End Delay & Worst performance & Better than AODV & Best performance & Better than AODV \\
\hline
\end{tabular}

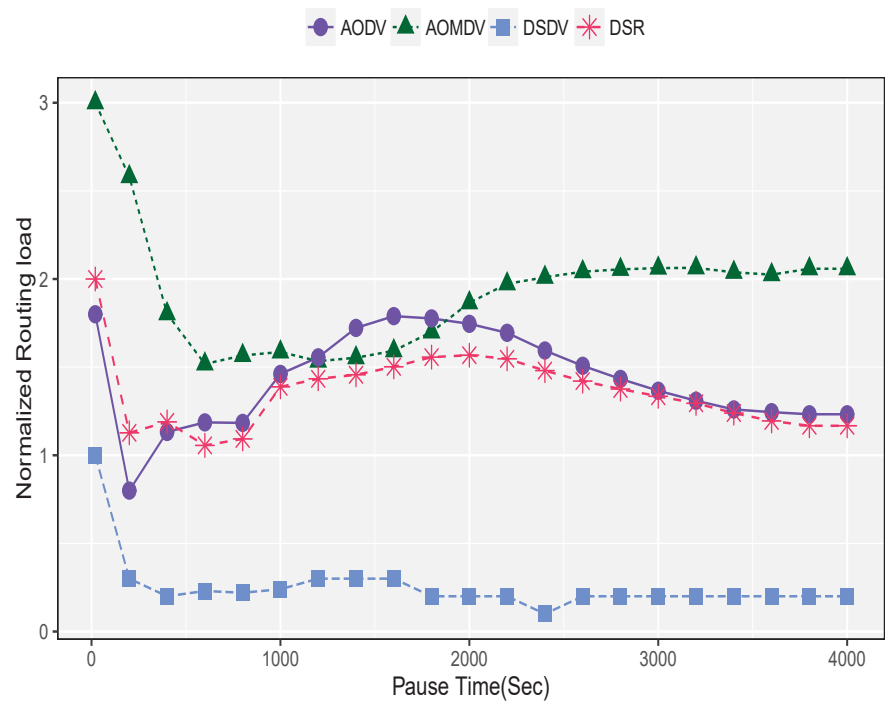

Fig. 4. Normalized Routing Load VS. Pause Time

Figure 4 shows the Normalized Routing Load vs pause time. AOMDV protocol represents the highest routing Load values due to the high number of route requests initiated while AODV and DSR showed approximately the same figure in contrast to the DSDV protocol which has the lowest routing load. Therefore, it can be inferred that DSDV represents the highest routing efficiency in terms of routing cost while AOMDV represents the highest routing performance in terms of packet delivery ratio. This gives a clear indication that the best packet delivery ratios are usually accompanied with the high routing load.

\section{B. End-to-end Delay}

Indicates the time it takes the packet to travel from the CBR source to reach the destination, it includes the delay that is caused by the route discovery process and the queue in the transmission of the data packet. Only the data packets that are successfully delivered to the destinations are counted as in equation (2).

$$
\text { EndToEndDelay }=\frac{\sum \text { Arrivetime }- \text { Sendtime }}{\sum \text { Numberofconnections }}
$$

Figure 5 represents the end to end delay for the four protocols in (milliseconds) vs pause time. It can be observed

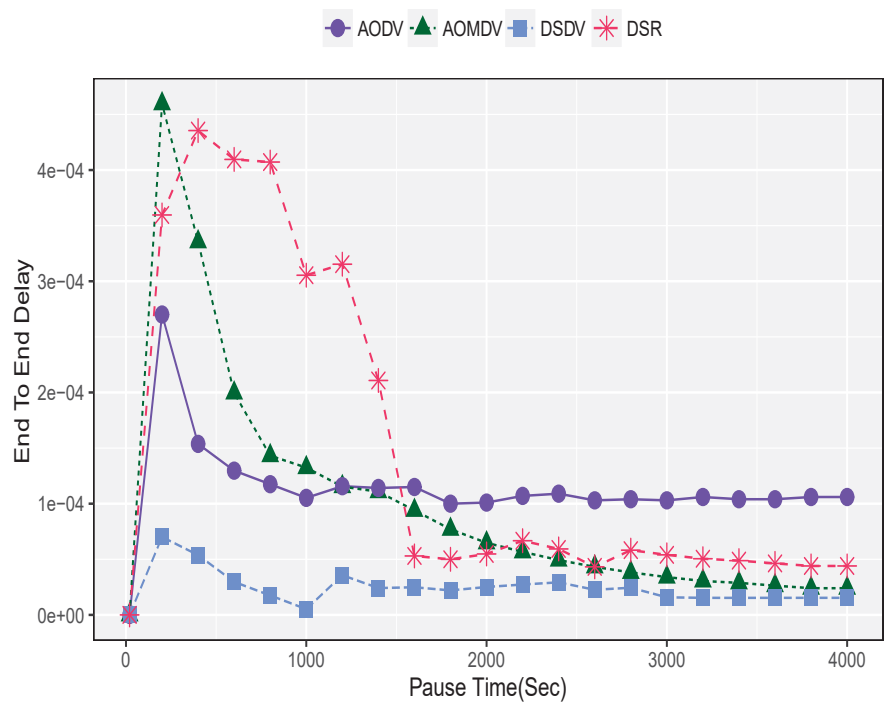

Fig. 5. End To End Delay VS. Pause Time

that AOMDV and DSR protocols show the largest initial delay reaching about 0.00045 milliseconds, which is necessary for route set up. While DSDV exhibits the lowest delay reaching only 0.00005 milliseconds which can be attributed to the fact that DSDV is a table driven protocol that maintains route tables that minimize the time needed for route discovery, while all the other protocols have on demand route discovery.

\section{Packet delivery ratio}

It is the ratio of data packets that arrive successfully to the destination, and can be calculated as in equation (3).

$$
P D R=\frac{\sum \text { Numberofpacketreceive }}{\sum \text { Numberofpacketsend }}
$$

In mobile Ad-Hoc networks, packet drop rate occurs due to transmission errors, mobility and congestion. Transmission errors are affected by the physical condition of the channel. The number of dropped packets in this environment (marine network) is very high. The effect of sparsity in the network is the main cause for this. Ships can travel for long distances without being in contact range and therefore unable to engage in the Ad-Hoc network using the available physical network. Figure 6 shows the comparison of packet delivery rate for AOMDV, AODV, DSDV and DSR protocol. DSDV produced 
the lowest packet delivery ratio. The maximum PDR reached almost $60 \%$ in AOMDV protocol.

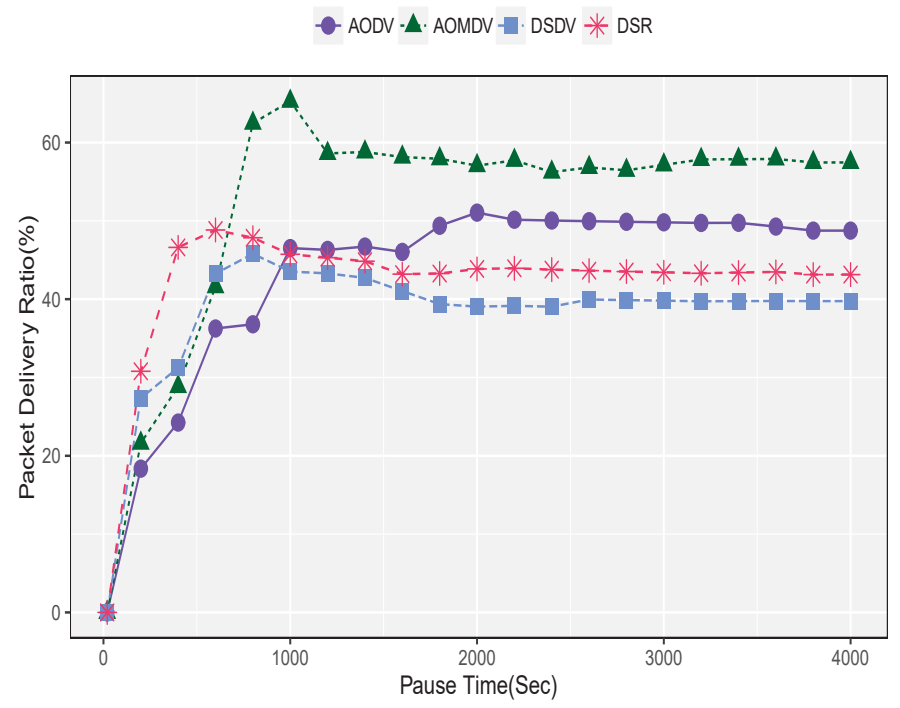

Fig. 6. Packet Delivery Ratio vs. Pause Time

\section{CONCLUSION}

Due to increased shipping and the high cost of other available technologies, the demand for data networks in the marine environment for cartography, safety and convenience shows an increasing trend. In this paper, implementing IoT data networks in a marine environment has been shown using the existing VHF communication infrastructure available on all ships. A model of the VHF radio that complies with the the International Telecommunication Union (ITU) standards for data communication has been used. The novel IoT cartographic application of localized and aggregated real-time sea maps has also been shown using low cost SANET networks.

\section{ACKNOWLEDGMENT}

This work was supported in part by the UK Engineering and Physical Sciences Research Council EPSRC EP/P016006/1.

\section{REFERENCES}

[1] B. Hui, K. Jeon, K. Chang, S. Kim, J. Park, and Y. Lim, "Design of radio transmission technologies for vhf band ship ad-hoc network," in ICT Convergence (ICTC), International Conference on. IEEE, 2011, pp. 626-629.

[2] S.-G. Kim, S. Sung, C. Yun, and Y.-K. Lim, "Design and implementation of a $\pi / 4$-dqpsk transmitter for maritime vhf digital communications," in OCEANS, 2011 IEEE-Spain. IEEE, 2011, pp. 1-5.

[3] M. Manoufali, H. Alshaer, P.-Y. Kong, and S. Jimaa, "Technologies and networks supporting maritime wireless mesh communications," in Wireless and Mobile Networking Conference (WMNC), 6th Joint IFIP. IEEE, 2013, pp. 1-8.

[4] R. Mohsin and J. Woods, "Performance evaluation of manet routing protocols in a maritime environment," in Computer Science and Electronic Engineering Conference (CEEC), 2014 6th. IEEE, 2014, pp. 1-5.

[5] R. J. Mohsin, J. Woods, and M. Q. Shawkat, "Density and mobility impact on manet routing protocols in a maritime environment," in Science and Information Conference (SAI), 2015. IEEE, 2015, pp. 1046-1051.
[6] M. Rabab J., W. John, and M. Q. Shawkat, “(amdc) algorithm for wireless sensor networks in the marine environment," International Journal of Advanced Computer Science and Applications(IJACSA), vol. 6, no. 6, 2015.

[7] A. K. Gupta, H. Sadawarti, and A. K. Verma, "Performance analysis of aodv, dsr \& tora routing protocols," IACSIT international journal of Engineering and Technology, vol. 2, no. 2, pp. 226-231, 2010.

[8] S. Mohseni, R. Hassan, A. Patel, and R. Razali, "Comparative review study of reactive and proactive routing protocols in manets," in Digital Ecosystems and Technologies (DEST), 2010 4th IEEE International Conference on. IEEE, 2010, pp. 304-309.

[9] M. Abolhasan, T. Wysocki, and E. Dutkiewicz, "A review of routing protocols for mobile ad hoc networks," Ad hoc networks, vol. 2, no. 1, pp. 1-22, 2004.

[10] N. Beijar, "Zone routing protocol (zrp)," Networking Laboratory, Helsinki University of Technology, Finland, vol. 9, pp. 1-12, 2002.

[11] M. Joa-Ng and I.-T. Lu, "A peer-to-peer zone-based two-level link state routing for mobile ad hoc networks," IEEE Journal on selected areas in communications, vol. 17, no. 8, pp. 1415-1425, 1999.

[12] S. Radhakrishnan, G. Racherla, C. N. Sekharan, N. S. Rao, and S. G. Batsell, "Dst-a routing protocol for ad hoc networks using distributed spanning trees," in Wireless Communications and Networking Conference, 1999. WCNC. 1999 IEEE, vol. 3. IEEE, 1999, pp. 1543-1547.

[13] N. Nikaein, H. Labiod, and C. Bonnet, "Ddr: distributed dynamic routing algorithm for mobile ad hoc networks," in Proceedings of the 1st ACM international symposium on Mobile ad hoc networking \& computing. IEEE Press, 2000, pp. 19-27. 